\title{
SIGNIFICANCE OF INTRA ANNUAL FLUCTUATIONS IN SOME SELECTED CONIFERS FROM A DRY TEMPERATE AREA (KALAM FOREST DIVISION), KHYBER PAKHTUNKHWA, PAKISTAN: A DENDROCHRONOLOGICAL ASSESSMENT
}

\author{
MuHAMmad, S. ${ }^{*}$ TAYYAB, M. - AKRAM, N. - MaliK, S. M. - AWAN, U. F. - KhAN, Z. - \\ HASNAIN, M. - ZAHID, M. - RASOOL, K. - KHAIRDIN, A. \\ Dendrochronology Lab., Department of Botany, Government College University, Lahore \\ 54000, Pakistan \\ *Corresponding author \\ e-mail:dr.sohaibmuhammad@gcu.edu.pk
}

(Received 28 $8^{\text {th }}$ Apr 2021; accepted 20 $0^{\text {th }}$ Aug 2021)

\begin{abstract}
Dendrochronological potential of some gymnosperms was determined by dividing a study site into 9 stands. 76 samples of Abies pindrow with maximum age of 698 years having $149.3 \mathrm{~cm}$ diameter, 23 samples of Taxus baccata with maximum age of 479 years having $137.2 \mathrm{~cm}$ diameter, 4 samples of Pinus roxburghii with maximum age of 218 years having 19.2 inches diameter and 2 samples of Cedrus deodara were obtained. All species were crossdated successfully by Skeleton Plot Model. Among them, mean growth of A. pindrow was $0.05-0.27 \mathrm{~cm}$ per year while for $T$. baccata it was $0.15-0.24 \mathrm{~cm}$ per year. Moreover, regression analysis between age and dbh was $(\mathrm{y}=0.0847 \mathrm{x}+4.0756),\left(\mathrm{R}^{2}=0.921\right)$ in $3^{\text {rd }}$ stand and $(\mathrm{y}=11.108 \mathrm{x}-41.174),\left(\mathrm{R}^{2}=0.8424\right)$ in $2^{\text {nd }}$ stand of A. pindrow and T. baccata respectively. The maximum value observed was in the $3^{\text {rd }}$ stand of T. baccata species which showed better correlation as compared to the rest of the stands. Strong correlation was also observed between TRW and difference of earlywood and latewood cell mass in all species. A. pindrow showed maximum value as $(\mathrm{y}=1.1397 \mathrm{x}+0.1873),\left(\mathrm{R}^{2}=0.9972\right)$.
\end{abstract}

Keywords: skeleton plot, Abies pindrow, Cedrus deodara, Pinus roxburghii, Taxus baccata

\section{Introduction}

Dendrochronology, "the study of tree time," is a multidisciplinary science that dates annual tree rings to their exact year formation to investigate prehistorical, historical and modern events (Palmer et al., 2011; Cook and Kairiukstis, 2010). It is applied in various subfields like climatology, ecology, forestry, fire history, geology, hydrology, volcanology and many other disciplines (Nash, 2002). Trees are intimately bound to environment as they record natural (temperature and precipitation) or unnatural (human induced) events or processes which can be seen in varying patterns of tree ring widths (Ali et al., 2021). Year to year climate variation induces variability in volume of wood that the tree produces in most geographic regions. When environmental conditions become favorable, trees respond by creating large volume of wood and produce less volume of wood in other years when conditions are unfavorable for growth (Sun et al., 2016; Panyushkina, 2011). Coniferous forests are important natural resources to sustain life in tropical, subtropical and temperate regions throughout the globe as they have economic and ecological importance. Among them, Kalam Forest Division (dry temperate area) is also geographically vulnerable to climate change due to environmental and some anthropogenic activities. The study was conducted in Kalam Forest division with objective to determine dendrochronological potential (age and 
growth rate studies) and to develop tree ring chronologies of conifers by SPM (Skeleton Plot Model) Method (Speer, 2010) (Fig. 1).

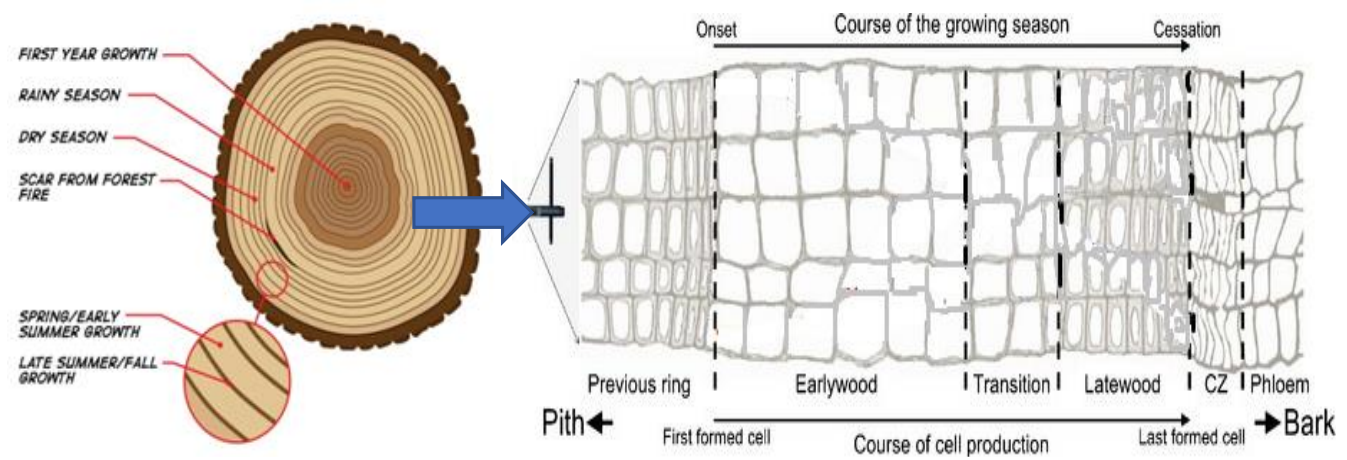

Figure 1. Annual ring fluctuations (earlywood and latewood) and anthropogenic behavior

\section{Chronology development by skeleton plot model}

Skeleton Plot Model is one of most appropriate and extensively used method by dendrochronologists to crossdate the tree samples which is the "procedure of matching ring width variations." Skeleton Plot, "Plot of vertical bars in which bar length is inversely related to tree ring width, is used to identify the double or false rings which occurs if seasonal growth is interrupted by severe climatic conditions, diseases or other agents and is later resumed a second growth layer, visible and added in during one growth season (Copenheaver et al., 2006). Such additional layer is false ring, which is simply a band of latewood cells between latewood part of previous ring and early wood part of next ring (Nash, 2002). As temperate trees (conifers) develop rings yearly so, patterns of tree ring widths are matched and compared in similar and dissimilar geologic regions (Vasconcellos et al., 2019; Speer, 2010).

Pakistan is more vulnerable to climate change due to environmental factors changing forest types (Bajwa et al., 2015) inducing health problems (Gosling et al., 2009), as well as reducing the oxygen levels in the sea (Shaffer et al., 2009). These changes are not uniform throughout globe and vary with change in regional temperature and precipitation so, dendrochronology helps to examine the past climate as well as to predict future climate by annual rings of trees (Shah et al., 2019). Many researchers have been engaged and are working in above mentioned areas. Muhammad et al. (2021) determined age and growth rate of pines. Ahmed and Naqvi (2005), Khan et al. (2008), Ahmed et al. (2009) determined dendrochronological potential of some gymnosperms from Swat, Dir, Chitral, Mansehra and Azad Kashmir, Pakistan. Khan (2011) determined dendrochronological potential of $C$. deodara and Pinus gerardiana. Wahab (2011) also estimated age and growth rates of conifers from Dir, Pakistan. Khan et al. (2013) developed tree ring chronologies and used in forest management, past climate investigation, wildfire and other hydrological aspects. The objectives of study were: (1) to estimate age and growth rate of selected conifers (A. pindrow, T. baccata, $P$. roxburghii and $C$. deodara); (2) to determine relationships, if any, between diameter/age, diameter/growth rate and between seasonal parts of annual rings of trees; (3) to determine correlation between different parameters to model meaningful relationships in form of regression analysis; (4) to develop Skeleton Plot Model of all 
samples, composite skeleton plot of climatically sensitive trees and their matching with master skeleton plots and raising their master chronologies.

\section{Study site}

Kalam Forest Division is geographically known as Swat Kohistan (Fig. 2), an independent Forest Division from last 5 decades as it was a part of Swat Forest Division in previous times. It consists of two tehsils known as Kalam and Behrain and covers an area about $3182.9272 \mathrm{~km}^{2}$. The site is climatically very sensitive as it lies in dry temperate zone, too much cold at high elevations causes migration of people to bottom of valley to fulfil their needs and survival. Here temperature and precipitation varies with altitude and appears in form of rain and snow. Rain falls from December to May as its average record is $492 \mathrm{~mm}$ and annual record is $423.56 \mathrm{~cm}$ (Iqbal, 2014).

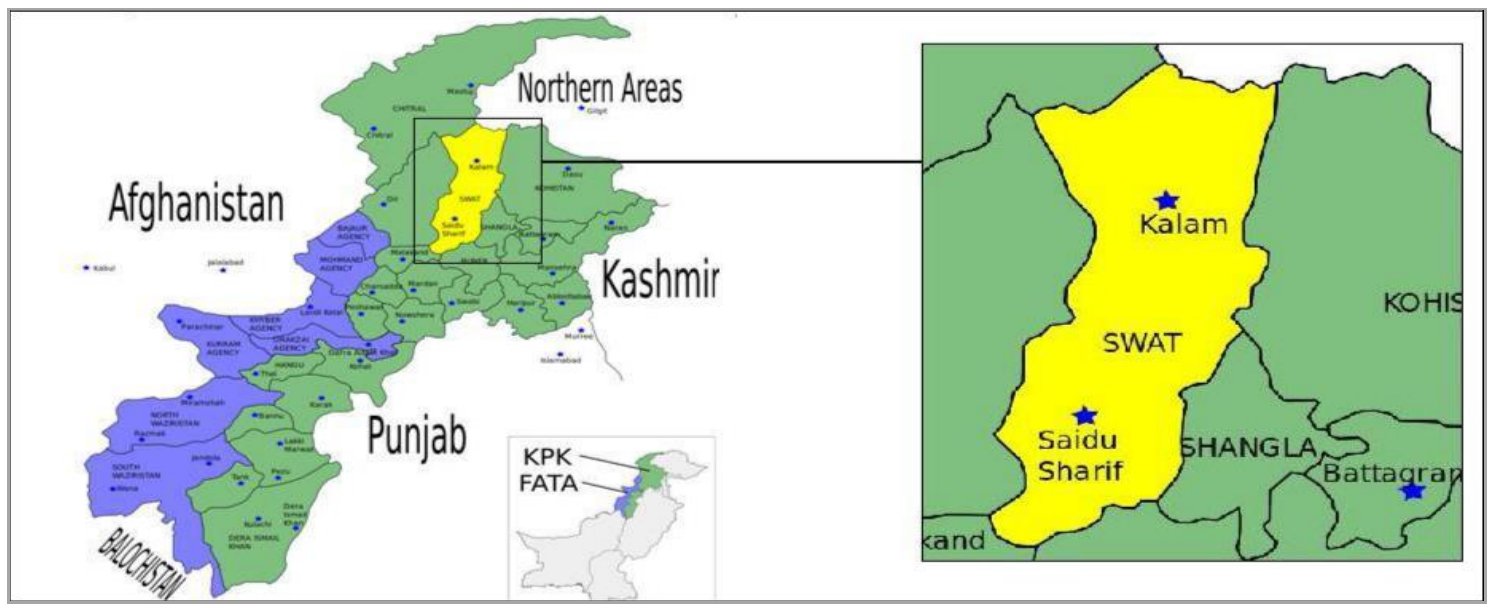

Figure 2. Divisional boundaries of Kalam Forest Division

\section{Materials and methods}

\section{Sample collection, processing and measurement}

Four conifer species were targeted for sample collection namely $A$. pindrow, $T$. baccata, $P$. roxburghii and $C$. deodara (Fig. 3). Subjective sampling was performed in the field and those individual trees were selected that were present on high and low elevation sites, steep slopes and well drained soils because they could possess the tree rings significantly sensitive to regional climate and cross dating could be performed successfully (Ahmed, 2014). Cores were obtained from healthy, rigid and unbranched trees by Swedish increment borers at height as $1.3 \mathrm{~m}$ or $4.3 \mathrm{ft}$. A total of 105 cores were obtained from 51 different trees in 2019. The cores were preserved in plastic straws to maintain alignment of cores. The ends of straws were covered with paper tape and holes created in trees were refilled by wax to protect them from any fungal or pathogen disease (Wahab et al., 2008). The diameter at breast height (dbh) of each tree was measured by dbh measuring tape (Hart and Grissino-Mayer, 2008). Later on, cores were mounted on wooden frames with glue and were allowed to dry. Sander machine fitted with different grades of sand papers $(80,100,120,150,180,320$ and 400 grit, depending upon particular species) was used to make smooth and fine surface of cores. It was proceeded until suitable polished surface was achieved after varnish coat (Phipps, 
1985). Velmex measuring system (TA4021H1) having connection with computer installed with $\mathrm{J} 2 \mathrm{X}$ software was used. All samples were measured one by one with respect to earlywood, latewood and total ring width (Volney and Mallet, 1992; Yamaguchi, 1991). All samples were crossdated successfully by skeleton plot method and further statistical analysis was performed.

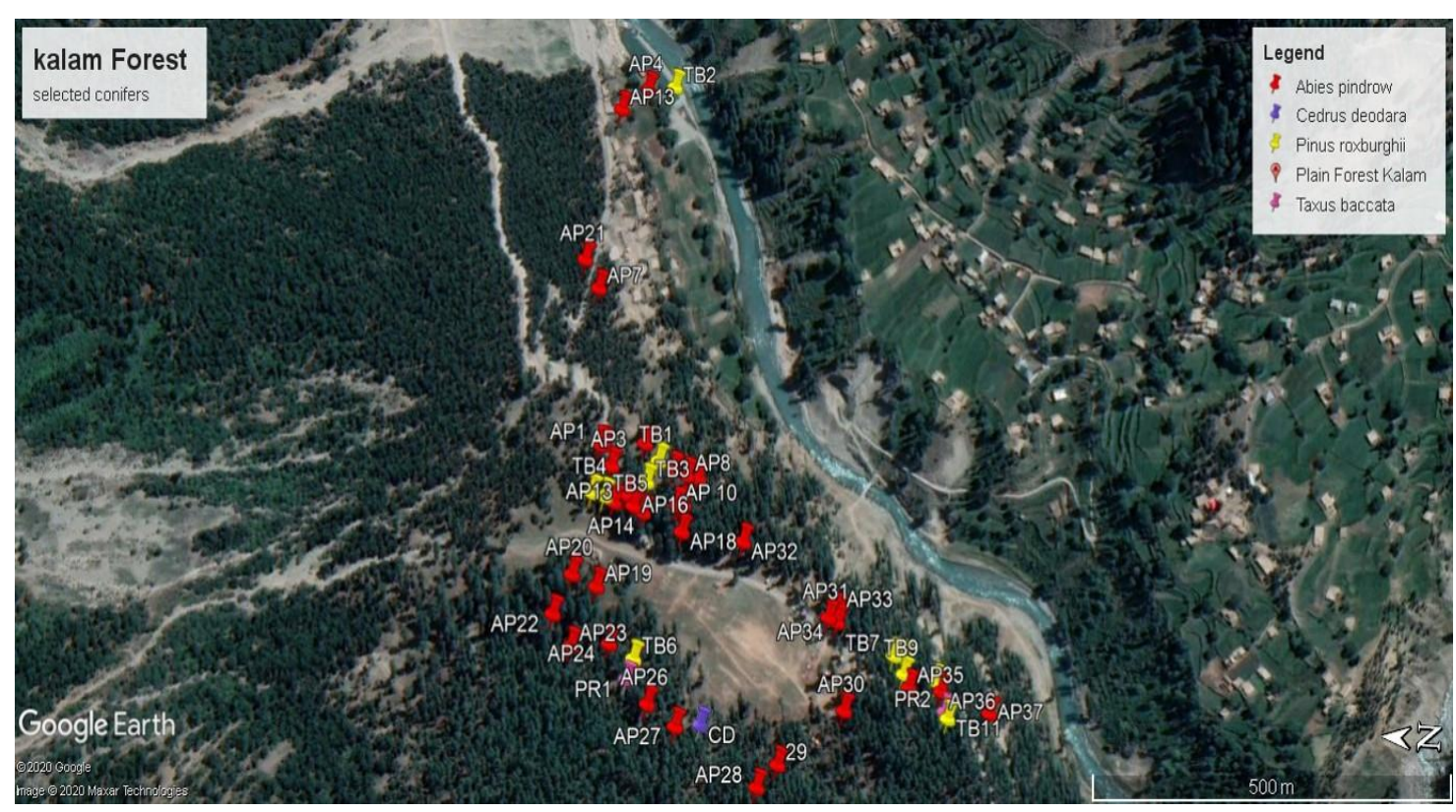

Figure 3. GPS points of selected conifers location in Kalam Forest Division KP, Pakistan

\section{Results}

Dendrochronological potential of above-mentioned species was determined by dividing study site into different stands and their relationships were determined by different parameters.

\section{Age and growth rate determination}

Age of all trees was estimated from nine stands of study site as shown in Table 1. The oldest tree was A. pindrow with 698 years age and $149.3 \mathrm{~cm}$ diameter. The youngest tree was 63 years of age with $73.15 \mathrm{~cm}$ diameter. An age of 479 years with $137.2 \mathrm{~cm}$ diameter was recorded in $T$. baccata as maximum age in this particular species while youngest was 113 years old having $45.72 \mathrm{~cm}$ diameter. In case of $P$. roxburghii, the maximum and minimum age was estimated as 218 years and 68 years with $48.76 \mathrm{~cm}, 67.05 \mathrm{~cm}$ diameter respectively. $C$. deodara was found to be minimal as regional climate and other topographic features of site were found to be unfavorable for this particular species.

Growth rate of all trees was also determined from this dry temperate area as shown in respective table. The maximum and minimum growth rate of $A$. pindrow was $0.48 \mathrm{~cm} /$ year and $0.05 \mathrm{~cm} /$ year. It was maximum as $0.06 \mathrm{~cm} /$ year in case of $T$. baccata while minimum was $0.12 \mathrm{~cm} /$ year. $P$. roxburghii also showed maximum and minimum growth rate as $0.15 \mathrm{~cm} /$ year and $0.10 \mathrm{~cm} /$ year respectively. Overall, T. baccata was found to be denser in study site and its growth rate was also recorded as maximum as $0.66 \mathrm{~cm} /$ year. 
Table 1. Age and growth rate studies of conifers

\begin{tabular}{c|c|c|c|c|c|c|c|c|c|c}
\hline \multirow{2}{*}{ Species } & \multicolumn{2}{|c|}{$\begin{array}{c}\text { Earlywood } \\
(\mathbf{m m})\end{array}$} & \multicolumn{2}{c|}{$\begin{array}{c}\text { Latewood } \\
(\mathbf{m m})\end{array}$} & \multicolumn{2}{c|}{$\begin{array}{c}\text { Age } \\
(\text { years })\end{array}$} & \multicolumn{2}{c|}{$\begin{array}{c}\text { Mean growth } \\
(\mathbf{m m})\end{array}$} & \multicolumn{2}{c}{$\begin{array}{c}\text { Growth rate } \\
\text { (cm/year) }\end{array}$} \\
\cline { 2 - 11 } & Max. & Min. & Max. & Min. & Max. & Min. & Max. & Min. & Max. & Min. \\
\hline A. pindrow & 4.7 & 0.55 & 0.53 & 0.13 & 698 & 63 & 0.16 & 0.02 & 0.48 & 0.05 \\
T. baccata & 6.5 & 1.22 & 0.71 & 0.14 & 479 & 113 & 0.24 & 0.06 & 0.66 & 0.12 \\
P. roxburghii & 1.45 & 1 & 0.24 & 0.14 & 218 & 68 & 0.05 & 0.04 & 0.15 & 0.10 \\
\hline
\end{tabular}

\section{Seasonal dynamics}

Tree ring width (TRW) and its parts (earlywood and latewood) were also measured (Fig. 1). The maximum TRW measurement was $4.86 \mathrm{~mm}$ and $0.69 \mathrm{~mm}$ as minimum in case of $A$. pindrow. The early and latewood part was $4.70 \mathrm{~mm}, 0.53 \mathrm{~mm}$ and $0.53 \mathrm{~mm}$, $0.13 \mathrm{~mm}$ respectively. In case of $T$. baccata, TRW was $6.64 \mathrm{~mm}$ and $1.37 \mathrm{~mm}$ as maximum and minimum. The early and latewood part was $6.5 \mathrm{~mm}, 1.2 \mathrm{~mm}$ and $0.71 \mathrm{~mm}, 0.41 \mathrm{~mm}$ respectively $P$. roxburghii showed TRW value as $1.66 \mathrm{~mm}$ and $1.11 \mathrm{~mm}$ and early latewood parts were $1.45 \mathrm{~mm}, 1.00 \mathrm{~mm}$ and $0.24 \mathrm{~mm}, 0.14 \mathrm{~mm}$ wide respectively.

\section{Correlations between dbh/age, dbh/growth rate, TRW and difference of earlywood, latewood cell mass}

Correlations of all species were determined (Table 2). Diameter at breast height (dbh) of A. pindrow showed positive significant correlation with age (Fig. $4 a$ ) while it was positive/negative correlated with growth rate (Fig. 4b). T. baccata showed positive correlation between dbh and age (Fig. 5a) and negative between dbh and growth rate (Fig. 5b) and it was found to be highly negative in P. roxburghii trees (Fig. 6a, b). Moreover, the correlation was also observed between tree ring width and difference of early, latewood cell mass. It was observed highly positive in all selected species gymnosperms (Figs. 4c, 5c, 6c). The maximum value was observed in $5^{\text {th }}$ stand of $A$. pindrow (Fig. 4c).

Table 2. Correlation and regression analysis between dbh/age, dbh/growth rate and TRW/early \& latewood cell mass

\begin{tabular}{c|c|c|c}
\hline Species & Parameter & Correlation $\left(\mathbf{R}^{2}\right)$ & Regression \\
\hline \multirow{3}{*}{ A. pindrow } & Dbh/age & 0.921 & $\mathrm{y}=0.0847 \mathrm{x}+4.0756$ \\
& Dbh/growth rate & 0.4126 & $\mathrm{y}=-339.62 \mathrm{x}+84.091$ \\
& TRW/Difference of earlywood and latewood cell mass & 0.9972 & $\mathrm{y}=1.1397 \mathrm{x}+0.1873$ \\
\hline \multirow{3}{*}{ T. baccata } & Dbh/age & 0.8424 & $\mathrm{y}=11.108 \mathrm{x}-41.174$ \\
& Dbh/growth rate & 0.4055 & $\mathrm{y}=-134.69 \mathrm{x}+55.873$ \\
& TRW/Difference of earlywood and latewood cell mass & 0.9646 & $\mathrm{y}=0.9417 \mathrm{x}+0.8654$ \\
\hline \multirow{3}{*}{ P. roxburghii } & Dbh/age & 0.6296 & $\mathrm{y}=-11.806 \mathrm{x}+414.67$ \\
& Dbh/growth rate & 0.0909 & $\mathrm{y}=-130.91 \mathrm{x}+29.018$ \\
& TRW/Difference of earlywood and latewood cell mass & 0.8943 & $\mathrm{y}=1.1081 \mathrm{x}+0.285$ \\
\hline
\end{tabular}



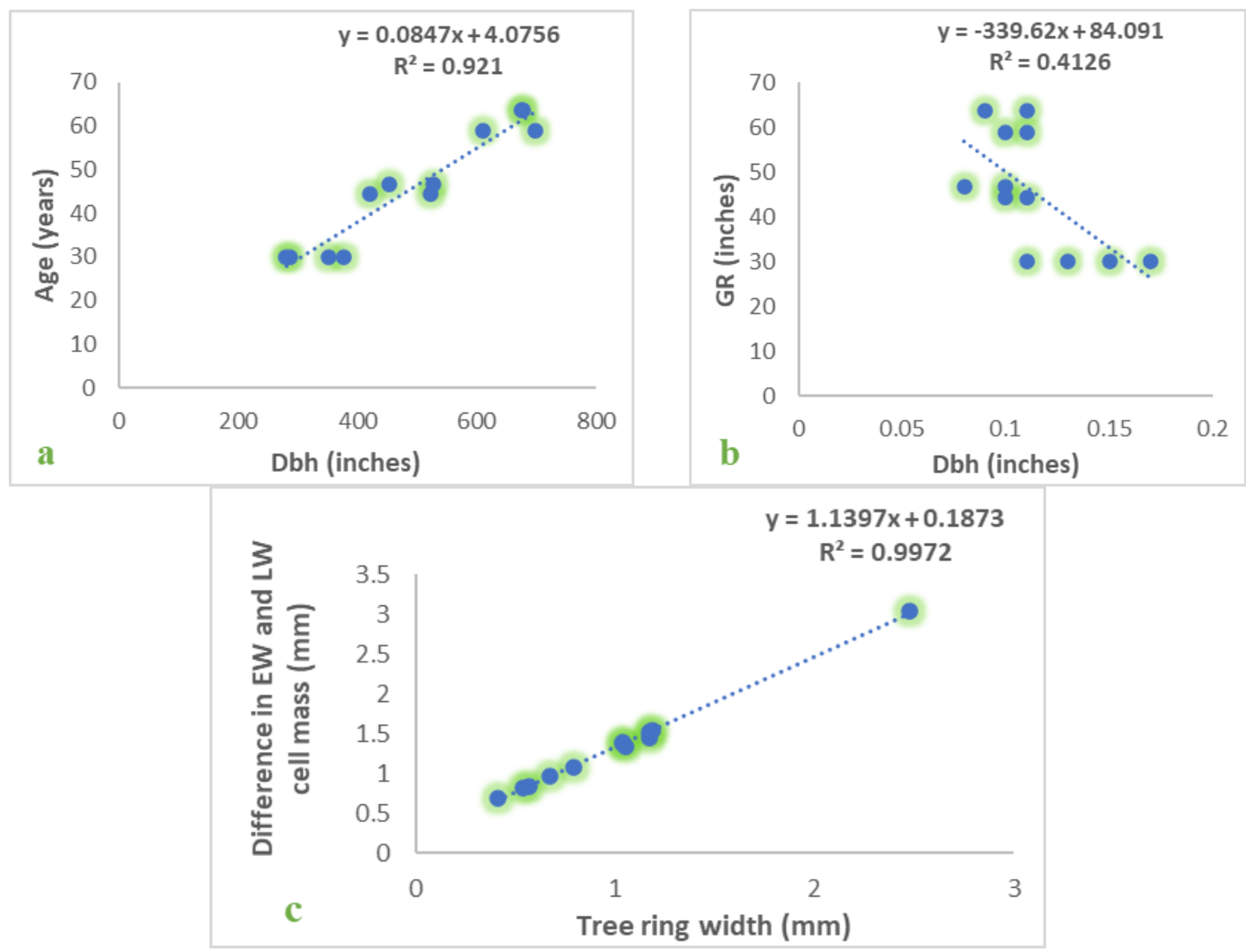

Figure 4. Correlation studies of A. pindrow

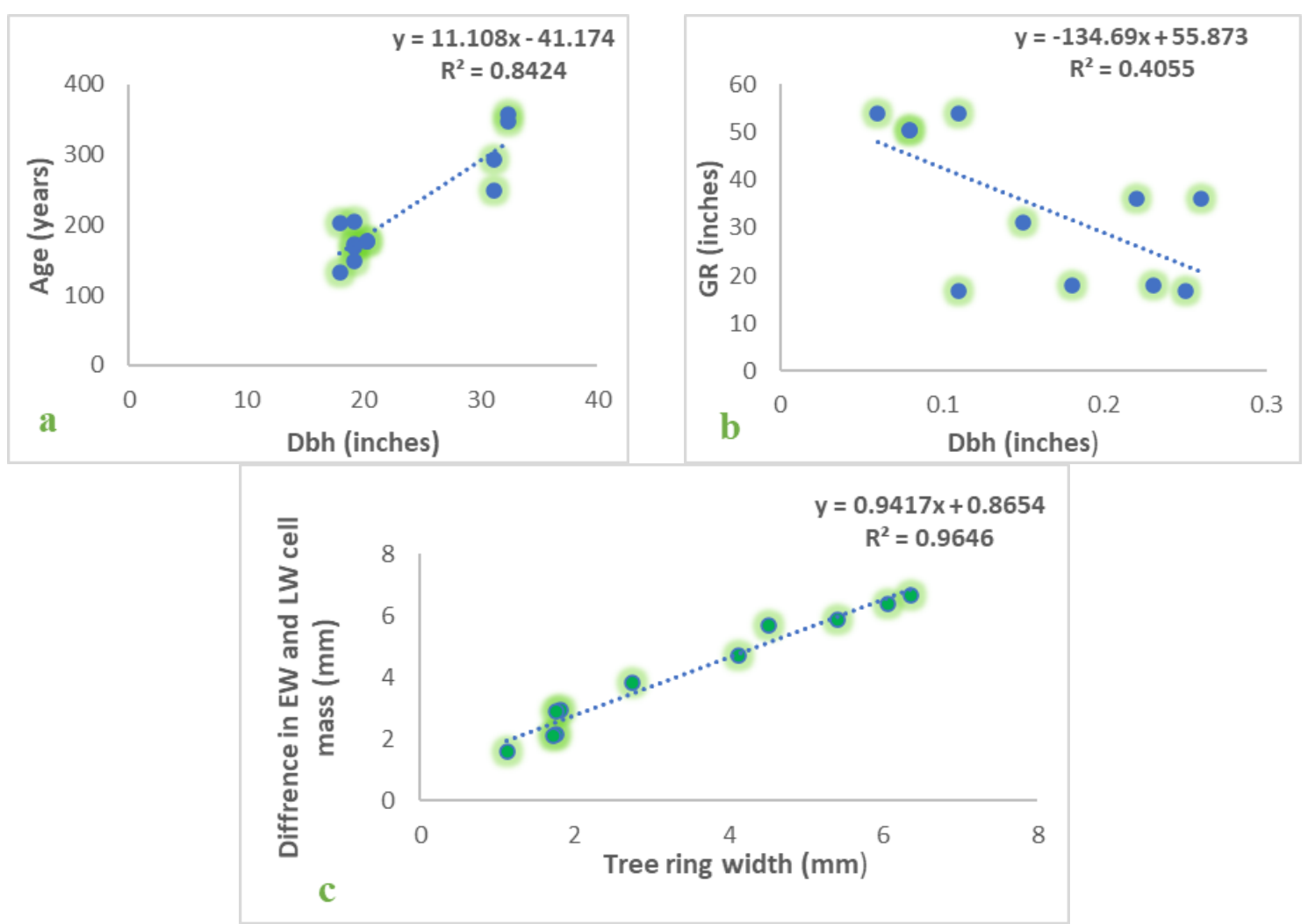

Figure 5. Correlation studies of T. baccata

APPLIED ECOLOGY AND ENVIRONMENTAL RESEARCH 19(6):4403-4419.

http://www.aloki.hu • ISSN 15891623 (Print) • ISSN 17850037 (Online)

DOI: http://dx.doi.org/10.15666/aeer/1906_44034419

(c) 2021, ALÖKI Kft., Budapest, Hungary 


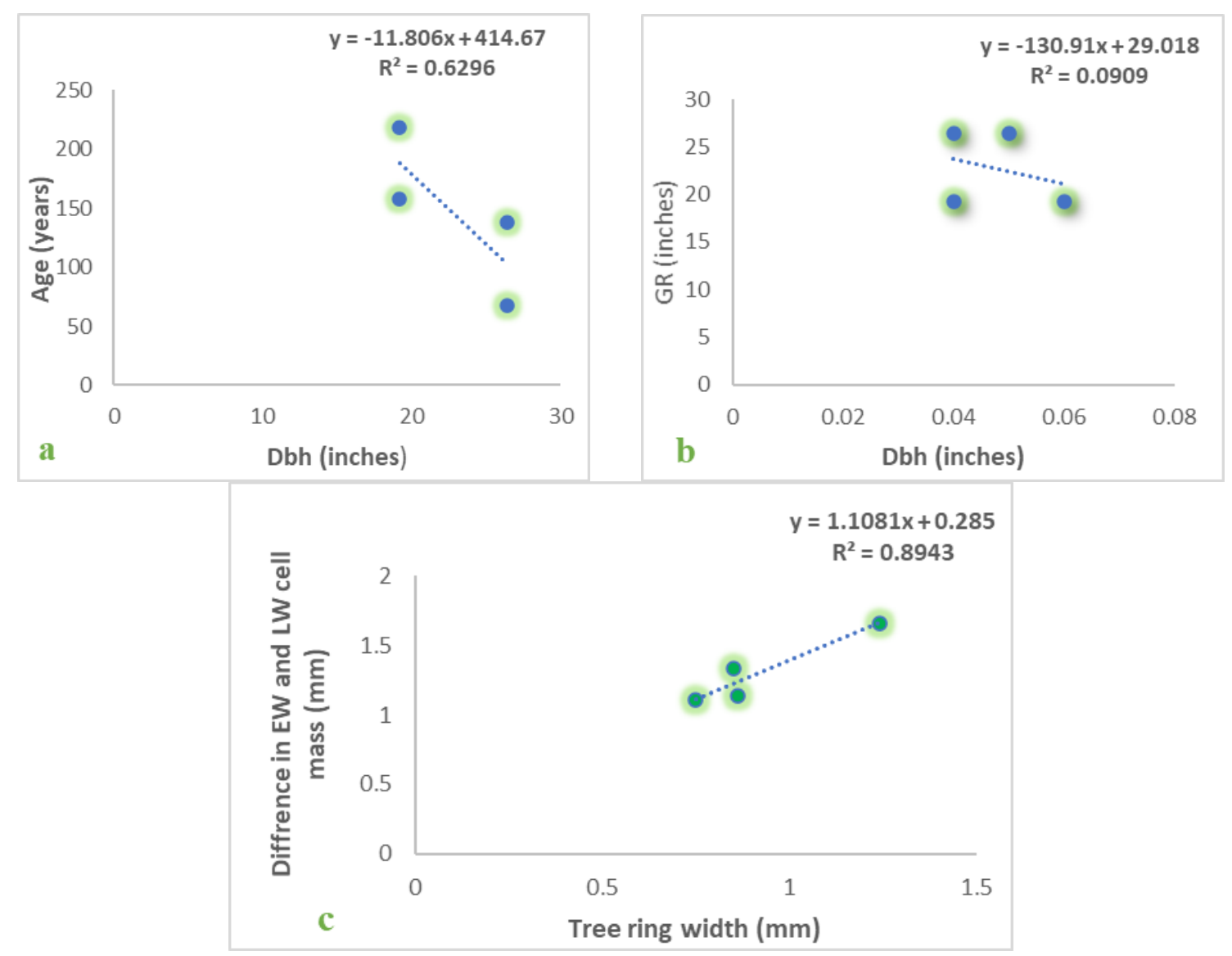

Figure 6. Correlation studies of $P$. roxburghii

\section{Skeleton plot model}

The fundamental principle of dendrochronology is crossdating which is simply crossmatching of tree ring width patterns among the species of similar and dissimilar geologic regions. It was applied to all trees either they showed complacent or sensitive growth patterns (Table 3). It was best represented by sensitive trees (Fig. 7) as they produced rings yearly or seasonally and responded well to climate fluctuations by creating larger and smaller rings while complacent trees produced rings of similar widths, as their growth was not affected by seasonal climate fluctuations. The samples showed sensitivity as they obtained from high and low elevation sites, steep slopes and well drained soils while others showed complacent nature as they were obtained from poor drained soils and near bank of river (Swat River, Pakistan).

Table 3. Tree ring matching patterns (chronology development) between samples of same trees through ***SPM method

\begin{tabular}{|c|c|c|c|c|c|}
\hline \multirow{2}{*}{ Sample codes } & \multirow{2}{*}{ Tree life span } & \multicolumn{2}{|c|}{ Matching pattern } & \multirow{2}{*}{$\begin{array}{l}\text { No. of matched } \\
\text { years }\end{array}$} & \multirow{3}{*}{$\begin{array}{c}\text { GPS } \\
\text { coordinates }\end{array}$} \\
\hline & & Narrow ring years & Wide ring years & & \\
\hline \multicolumn{5}{|c|}{ A. pindrow } & \\
\hline AP1 and AP1' & 1929-2019AD & 1998, 1999 & ---- & 2 & $\begin{array}{l}\mathrm{N}: 35.51123 \\
\mathrm{E}: 72.60368\end{array}$ \\
\hline AP2 and AP2' & 1888-2019AD & $\begin{array}{l}1905-1927,1933-1945,1947- \\
1974,1995-1998,2010-2019\end{array}$ & ---- & 78 & $\begin{array}{l}\mathrm{N}: 35.51118 \\
\mathrm{E}: 72.60368\end{array}$ \\
\hline
\end{tabular}




\begin{tabular}{|c|c|c|c|c|c|}
\hline AP3 and AP3' & 1926-2019AD & $\begin{array}{c}1967,1978,1984,1985,1991- \\
1993,2000,2010,2011\end{array}$ & ---- & 10 & $\begin{array}{l}\mathrm{N}: 35.51107 \\
\mathrm{E}: 72.60342\end{array}$ \\
\hline AP4 and AP4' & 1926-2019AD & $\begin{array}{c}1967,1968,1982,1983,1992, \\
2001-2003,2013,2018,2019\end{array}$ & ---- & 11 & $\begin{array}{l}N: 35.51095 \\
E: 72.60850\end{array}$ \\
\hline AP7 and AP7' & 1933-2019AD & $\begin{array}{l}1946,1964,1996,1997,2009, \\
2010\end{array}$ & $1977-1986$ & 16 & $\begin{array}{l}\mathrm{N}: 35.51068 \\
\mathrm{E}: 72.60374\end{array}$ \\
\hline AP11 and AP11' & 1817-2019AD & $\begin{array}{c}\text { 1931-1934, 1985-1989, 1994, } \\
2018,2019\end{array}$ & ---- & 12 & $\begin{array}{l}\mathrm{N}: 35.51031 \\
\mathrm{E}: 72.60343\end{array}$ \\
\hline AP12 and AP12' & 1897-2019AD & \begin{tabular}{|c|} 
1936-1938, 1981-1983, 1989, \\
2001-2003, 2005-2008, 2010-2019 \\
\end{tabular} & ---- & 24 & $\begin{array}{l}N: 35.51080 \\
E: 72.60860\end{array}$ \\
\hline AP13 and AP13' & 1819-2019AD & $\begin{array}{c}\text { 1944, 1953-1961, 2015, 2018, } \\
2019\end{array}$ & ---- & 13 & $\begin{array}{l}N: 35.51014 \\
E: 72.60337 \\
\end{array}$ \\
\hline AP14 and AP14' & 1846-2019AD & \begin{tabular}{|c|}
$1903,1904,1937,1938,1943-$ \\
$1946,1951-1974,2007-2014,2019$
\end{tabular} & $\begin{array}{c}1900,1901,1983- \\
1993,1998-2003 \\
\end{array}$ & 59 & $\begin{array}{l}N: 35.51000 \\
E: 72.60334\end{array}$ \\
\hline AP15 and AP15' & 1830-2019AD & $\begin{array}{c}\text { 1915-1924, 1936-1945, 1968- } \\
1973,1995-1997\end{array}$ & $1948,2005-2007$ & 33 & $\begin{array}{l}\mathrm{N}: 35.51024 \\
\mathrm{E}: 72.60297\end{array}$ \\
\hline AP16 and AP16' & 1857-2019AD & $\begin{array}{c}1879-1882,1889,1900,1907- \\
1912,1958-1965,1973-1987 \\
1990-1993\end{array}$ & $\begin{array}{l}1946,1947,2005- \\
2009\end{array}$ & 46 & $\begin{array}{l}N: 35.51020 \\
E: 72.60298\end{array}$ \\
\hline AP17 and AP17' & 1879-2019AD & $\begin{array}{l}\text { 1926-1945, 1973-1977, 1979- } \\
1985,1999,2000,2011-2019\end{array}$ & ---- & 43 & $\begin{array}{l}\mathrm{N}: 35.51016 \\
\mathrm{E}: 72.60300\end{array}$ \\
\hline AP18 and AP18' & 1930-2019AD & $\begin{array}{c}1983,1984,1986,1989-1991 \\
2001-2005,2018,2019\end{array}$ & $1982,1995-1999$ & 19 & $\begin{array}{l}N: 35.51127 \\
E: 72.60818 \\
\end{array}$ \\
\hline AP21 and AP21' & 1922-2019AD & $\begin{array}{c}\text { 1959-1963, 1975-1977, 1982, } \\
\text { 1983, 1998-2001, 2010-2012, } \\
\text { 2016-2019 }\end{array}$ & ---- & 21 & $\begin{array}{l}N: 35.51101 \\
E: 72.60296\end{array}$ \\
\hline AP22 and AP22' & 1942-2019AD & $1983,1998-2000,2016-2019$ & ---- & 8 & $\begin{array}{l}\mathrm{N}: 35.51086 \\
\mathrm{E}: 72.60307 \\
\end{array}$ \\
\hline AP23 and AP23' & 1897-2019AD & $\begin{array}{l}1940-1947,1952-1954,1982, \\
1983,1987-1989,2001-2019\end{array}$ & ---- & 35 & $\begin{array}{l}\mathrm{N}: 35.51077 \\
\mathrm{E}: 72.60293\end{array}$ \\
\hline AP24 and AP24' & 1910-2019AD & $\begin{array}{c}1948,1949,1975,1980,1998, \\
2018,2019\end{array}$ & ---- & 7 & $\begin{array}{l}N: 35.51065 \\
E: 72.60280\end{array}$ \\
\hline AP25 and AP25' & 1900-2019AD & $\begin{array}{c}1940,1947,1984,1967-1973, \\
1999,2011,2018,2019\end{array}$ & ---- & 14 & $\begin{array}{l}N: 35.51016 \\
E: 72.60265 \\
\end{array}$ \\
\hline AP26 and AP26' & 1859-2019AD & $\begin{array}{c}1926-1931,1933,1938-1942 \\
1944-1947,1949,2001,2009- \\
2015,2018,2019\end{array}$ & $1911-1924$ & 41 & $\begin{array}{l}\mathrm{N}: 35.51115 \\
\mathrm{E}: 72.60189\end{array}$ \\
\hline AP27 and AP27' & 1929-2019AD & 2001 & ---- & 1 & $\begin{array}{l}\mathrm{N}: 35.51145 \\
\mathrm{E}: 72.80202 \\
\end{array}$ \\
\hline AP28 and AP28' & 1859-2019AD & $\begin{array}{c}1904,1970,1975,1981-1986, \\
1996,2002-2007,2010-2019\end{array}$ & $\begin{array}{l}1936,1937,1998- \\
2001\end{array}$ & 32 & $\begin{array}{l}\mathrm{N}: 35.51158 \\
\mathrm{E}: 72.80608 \\
\end{array}$ \\
\hline AP29 and AP29' & 1879-2019AD & $1975,1976,2012-2019$ & ---- & 10 & $\begin{array}{l}N: 35.51164 \\
E: 72.60152\end{array}$ \\
\hline AP30 and AP30' & 1801-2019AD & $\begin{array}{c}1820-1828,1857-1865,1872- \\
1876,1929-1931,1945-1951, \\
1960,1961,1964-1966,1996- \\
1999,2002-2005,2009-2019\end{array}$ & $\begin{array}{c}1897-1903,1936 \\
1910-1914\end{array}$ & 70 & $\begin{array}{l}N: 35.51140 \\
E: 72.60114\end{array}$ \\
\hline AP31 and AP31' & 1872-2019AD & $\begin{array}{c}1911,1915,1916,1943-1953, \\
1959-1999\end{array}$ & $2005-2014$ & 65 & $\begin{array}{l}N: 35.51095 \\
E: 72.60119\end{array}$ \\
\hline AP32 and AP32' & 1679-2019AD & $1729-1755$ & $\begin{array}{c}1760-1767,1783- \\
1835,1837-2019 \\
\end{array}$ & 271 & $\begin{array}{l}\mathrm{N}: 35.51064 \\
\mathrm{E}: 72.60100\end{array}$ \\
\hline AP35 and AP35' & 1775-2019AD & \begin{tabular}{|c|}
$1838-1840,1844-1852,1856-$ \\
$1870,1881-1900,1906-1910$ \\
$1913-1956,1958,1961,1962$ \\
$1965,1973,1974,1976-1978$ \\
$1981-1992,1996-2004,2018,2019$ \\
\end{tabular} & & 128 & $\begin{array}{l}N: 35.51045 \\
E: 72.60047\end{array}$ \\
\hline AP36 and AP36' & 1806-2019AD & \begin{tabular}{|c|}
$1977-2004,2007,1881-1890$, \\
$1898-1951,1969-1974,2018,2019$ \\
\end{tabular} & $1953-1967$ & 115 & $\begin{array}{l}N: 35.51010 \\
E: 72.60021 \\
\end{array}$ \\
\hline AP38 and AP38' & 1834-2019AD & $\begin{array}{l}\text { 1907-1913, 1915, 1927-1930, } \\
1932-1945,1948-1953,1964, \\
1965,1974-1977,2002-2019\end{array}$ & ---- & 56 & $\begin{array}{l}N: 35.50907 \\
E: 72.59952\end{array}$ \\
\hline AP39 and AP39' & 1802-2019AD & $1930-1950,1983-2019,1964-1973$ & $1908-1918$ & 89 & $\begin{array}{l}\mathrm{N}: 35.50883 \\
\mathrm{E}: 72.59981 \\
\end{array}$ \\
\hline
\end{tabular}




$$
-4411 \text { - }
$$

\begin{tabular}{|c|c|c|c|c|c|}
\hline AP40 and AP40' & 1815-2019AD & 1977, 1980-1982, 2012, 2013 & 1977 & 7 & $\begin{array}{l}\mathrm{N}: 35.50805 \\
\mathrm{E}: 72.80054\end{array}$ \\
\hline AP41 and AP41' & 1833-2019AD & $\begin{array}{c}1946-1950,1960,1975,1976, \\
1984-1989,1987,1993-2000 \\
2012-2014\end{array}$ & ---- & 26 & $\begin{array}{l}N: 35.50829 \\
E: 72.60169\end{array}$ \\
\hline AP42 and AP42' & 1898-2019AD & 1934-1939 & --- & 6 & $\begin{array}{l}\mathrm{N}: 35.50940 \\
\mathrm{E}: 72.60258\end{array}$ \\
\hline AP43 and AP43' & 1944-2019AD & 1992-1999 & ---- & 8 & $\begin{array}{l}N: 35.50817 \\
E: 72.60177\end{array}$ \\
\hline AP44 and AP44' & 1903-2019AD & $1925-1949,1956-1962,1977-2005$ & 1976 & 62 & $\begin{array}{l}\mathrm{N}: 35.50818 \\
\mathrm{E}: 72.60164 \\
\end{array}$ \\
\hline AP48 and AP48' & 1891-2019AD & 1988-1994, 2008-2019 & ---- & 19 & $\begin{array}{l}N: 35.50685 \\
E: 72.60086\end{array}$ \\
\hline AP50 and AP50' & 1876-2019AD & 2019-1990, 1933, 1932 & ---- & 32 & $\begin{array}{l}N: 35.50980 \\
E: 72.60096\end{array}$ \\
\hline \multicolumn{6}{|c|}{ T. baccata } \\
\hline TB5 & 1953-2019AD & ---- & --- & --- & $\begin{array}{l}\mathrm{N}: 35.51085 \\
\mathrm{E}: 72.60382 \\
\end{array}$ \\
\hline TB6 and TB6' & 1918-2019AD & 1978,1979 & ---- & 2 & $\begin{array}{l}\mathrm{N}: 35.51063 \\
\mathrm{E}: 72.60875 \\
\end{array}$ \\
\hline TB8 and TB8' & 1985-2019 AD & --- & $\begin{array}{c}\text { 2016-2013, 2009, } \\
2001,2000,1999 \\
1998 \\
\end{array}$ & 9 & $\begin{array}{l}\mathrm{N}: 35.51050 \\
\mathrm{E}: 72.60353\end{array}$ \\
\hline TB9 and TB9' & 1950-2019AD & 2018,2019 & --- & 2 & $\begin{array}{l}N: 35.51061 \\
E: 72.60857\end{array}$ \\
\hline TB10 and TB10' & 1932-2019AD & $\begin{array}{c}1960,1961,1963,1964,2018, \\
2019\end{array}$ & $1954,1992,1993$ & 9 & $\begin{array}{l}\mathrm{N}: 35.51063 \\
\mathrm{E}: 72.60325 \\
\end{array}$ \\
\hline TB19 and TB19' & 1966-2019AD & 1998-2000, 2018, 2019 & 1993 & 6 & $\begin{array}{l}\mathrm{N}: 35.51128 \\
\mathrm{E}: 72.60306 \\
\end{array}$ \\
\hline TB20 and TB20' & 1953-2019AD & 1997, 2018, 2019 & ---- & 3 & $\begin{array}{l}\mathrm{N}: 35.51113 \\
\mathrm{E}: 72.60306 \\
\end{array}$ \\
\hline TB33 and TB33' & 1903-2019AD & 2019-1996, 1998, 1987 & --- & 24 & $\begin{array}{l}\mathrm{N}: 35.51113 \\
\mathrm{E}: 72.60303\end{array}$ \\
\hline TB45 and TB45' & 1894-2019AD & $\begin{array}{c}\text { 1951, 1971-1982, 1989-1995, } \\
\text { 2008-2011, 2016-2019 }\end{array}$ & --- & 28 & $\begin{array}{l}\mathrm{N}: 35.51065 \\
\mathrm{E}: 72.60102 \\
\end{array}$ \\
\hline TB46 and TB46' & 1899-2019AD & $\begin{array}{c}1972,1973,1979,1980,1996- \\
2000\end{array}$ & 2006-2008 & 12 & $\begin{array}{l}N: 35.51065 \\
E: 72.60102 \\
\end{array}$ \\
\hline TB47 and TB47' & 1859-2019AD & 1904-1909, 1932-1934, 1960-1966 & $1998-2019$ & 38 & $\begin{array}{l}\mathrm{N}: 35.50746 \\
\mathrm{E}: 72.60127\end{array}$ \\
\hline TB49 and TB49' & 1874-2019AD & \begin{tabular}{|c|}
$1938-1940,1956,1992,1993$, \\
$1997,1998,2004-2008,2010-2019$
\end{tabular} & 1970 & 24 & $\begin{array}{l}\mathrm{N}: 35.50734 \\
\mathrm{E}: 72.60123 \\
\end{array}$ \\
\hline \multicolumn{6}{|c|}{ P. roxburghii } \\
\hline PR34 and PR34' & 1814-2019AD & $\begin{array}{l}\text { 1827, 1828, 1918-1923, 1929, } \\
\text { 1932-1939, 1940, 1942, 1943, } \\
\text { 1992, 1993, 1998-2000, 2007, } \\
\text { 2008, 2018, } 2019\end{array}$ & $1887-1906$ & 49 & $\begin{array}{l}N: 35.51072 \\
E: 72.60047\end{array}$ \\
\hline PR51 and PR51' & 1877-2019AD & 1985-1988, 2004-2019 & 1995-1998 & 24 & $\begin{array}{l}N: 35.50680 \\
E: 72.60061\end{array}$ \\
\hline \multicolumn{6}{|c|}{ C. deodara } \\
\hline CD37and CD37' & 1783-2019AD & $\begin{array}{c}\text { 1860-1864, 1905, 1932-1936, } \\
\text { 1945-1947, 1949, 1951-1954, } \\
\text { 1959-1960, 1970-1988, 1998- } \\
\text { 2010, 2013-2019 }\end{array}$ & ---- & 60 & $\begin{array}{l}\mathrm{N}: 35.50980 \\
\mathrm{E}: 72.60024\end{array}$ \\
\hline
\end{tabular}

After crossdating all the core samples, composite skeleton plots of the most sensitive trees were made between two cores of each individual tree which highlighted the most sensitive years of growth. These plots were matched with the master skeleton plot and master chronology was developed as shown in Figure $7 a-h$.

Composite skeleton plots, master skeleton plots and master chronologies of sensitive trees were presented in Figure 7. 


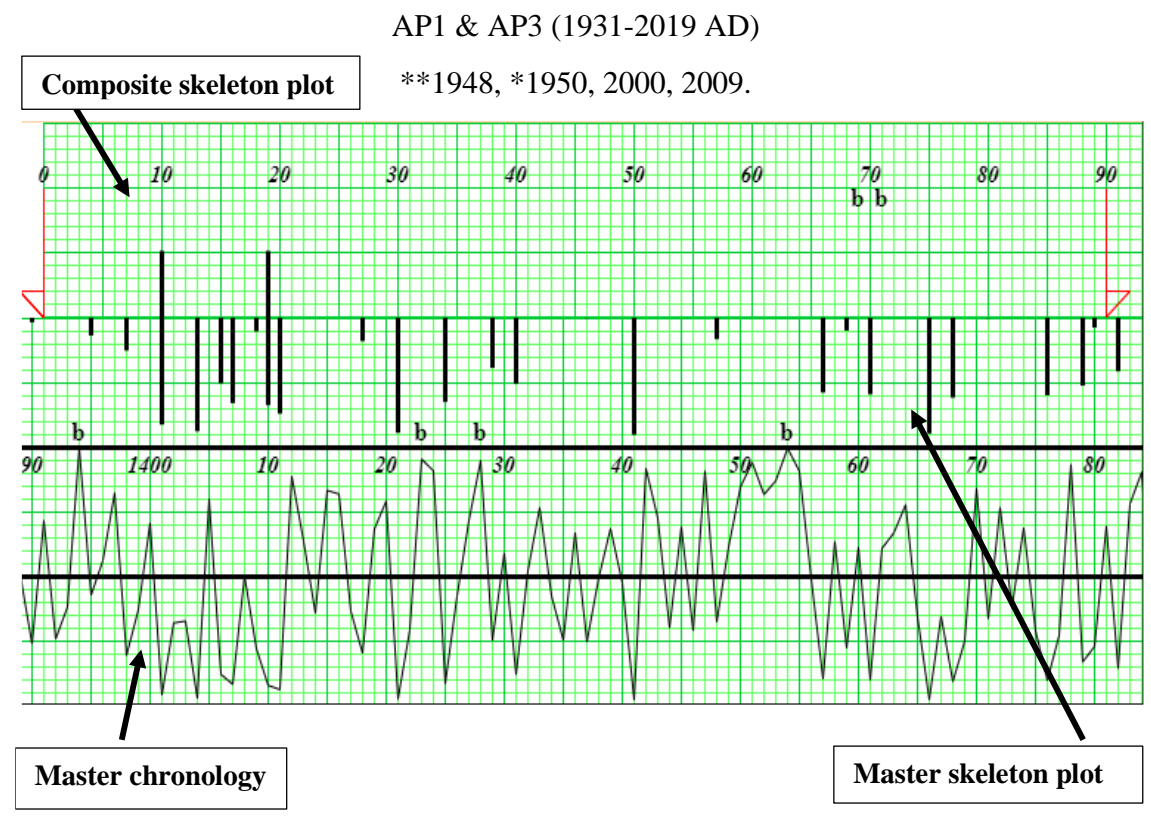

a

AP4 \& AP7 (1928-2019 AD)

*1934-1937, *1940, 1948, 2010, 2009

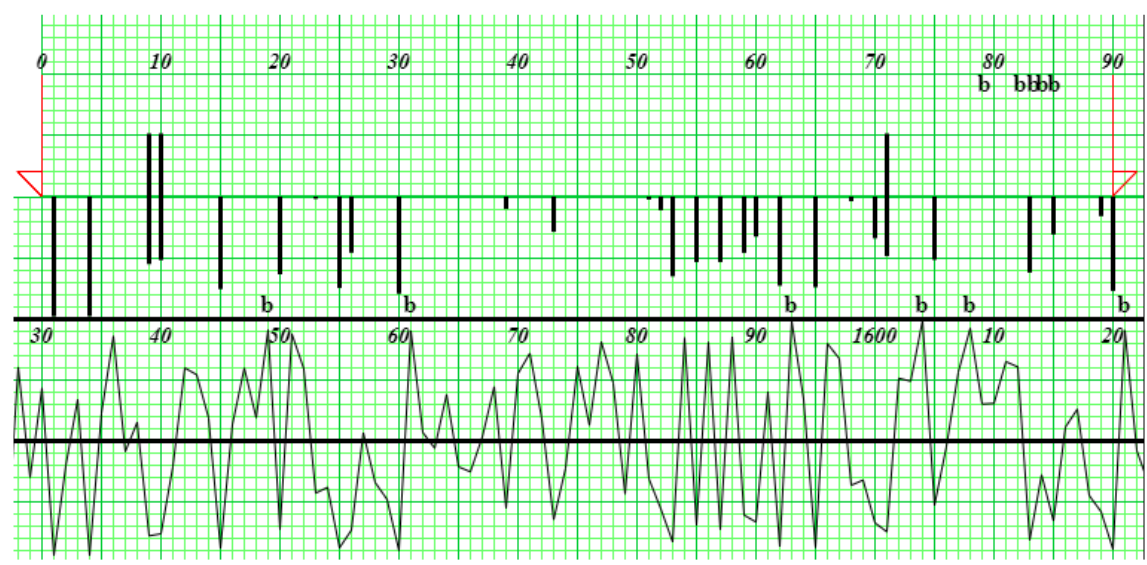

b

AP12 \& AP13' (1897-2019 AD)

1957-1961, 1976, 1978, 1983, 1987, 1988, 2015, 2018.

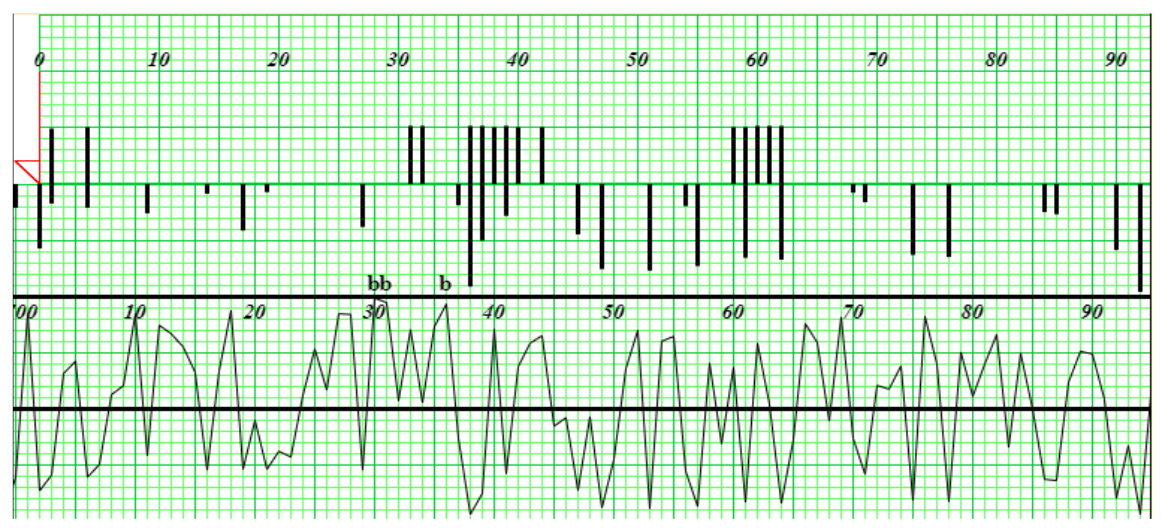

c 


$$
-4413 \text { - }
$$

AP15 \&AP18' (1887-2019 AD)

*1956, 1979-1981, 2000-2003.

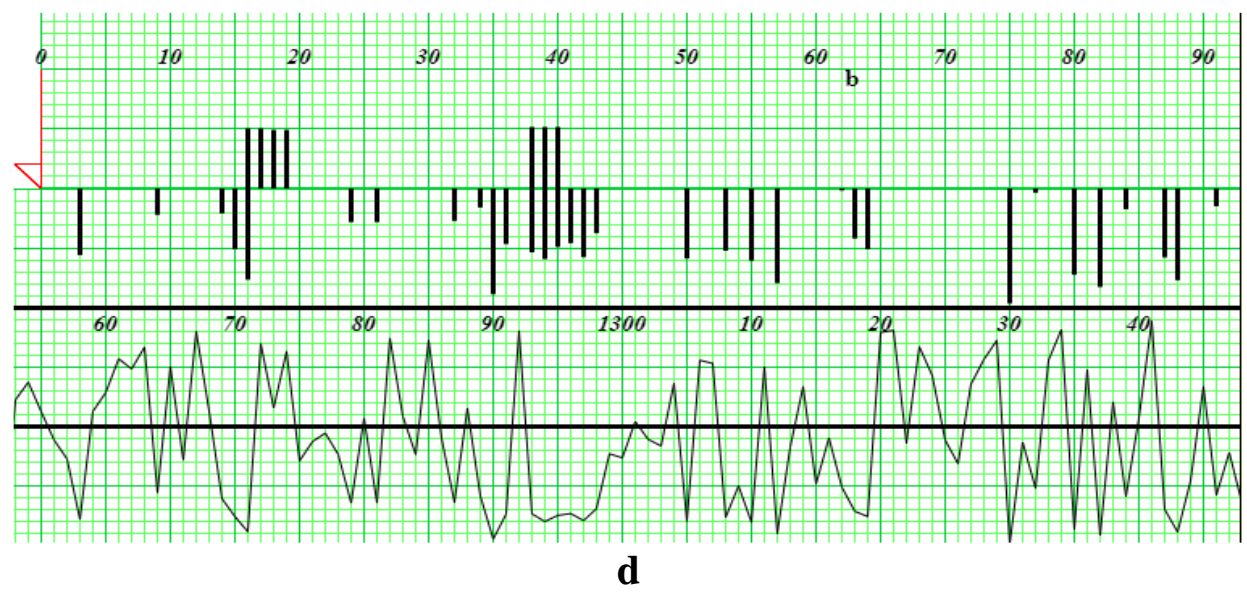

AP22 \& AP24 (1911-2019 AD)

$*(1958,1959,1961), 1975,1983,1998,2017,2018$.

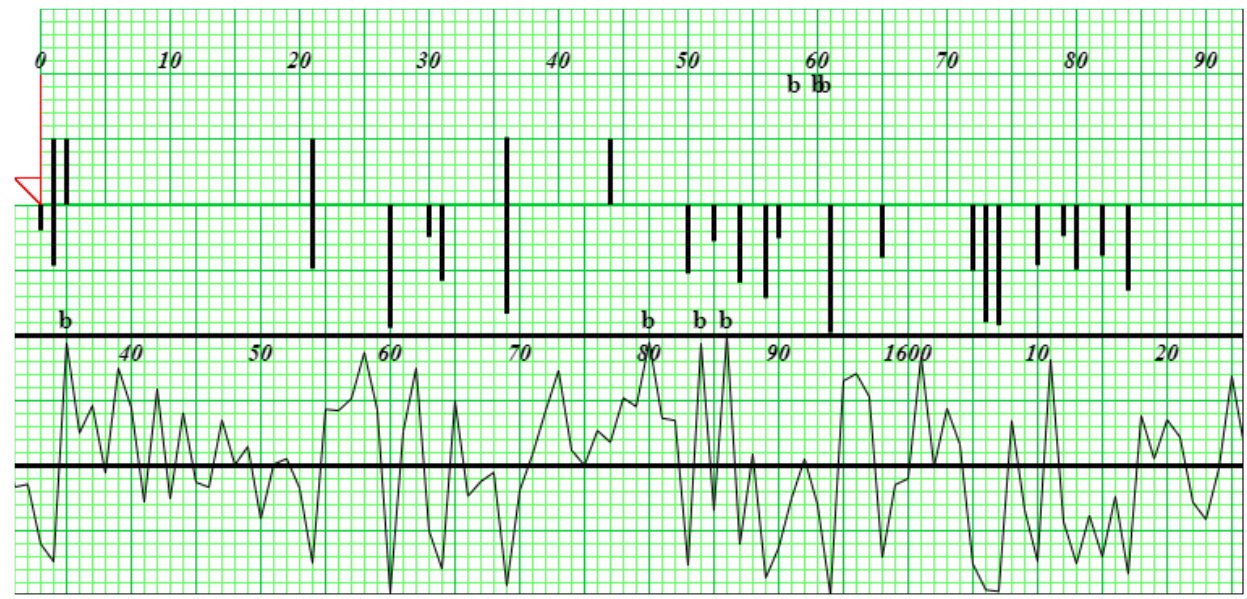

$\mathbf{e}$

AP27 \& AP42’ (1898-2019 AD)

*1967, 2001.

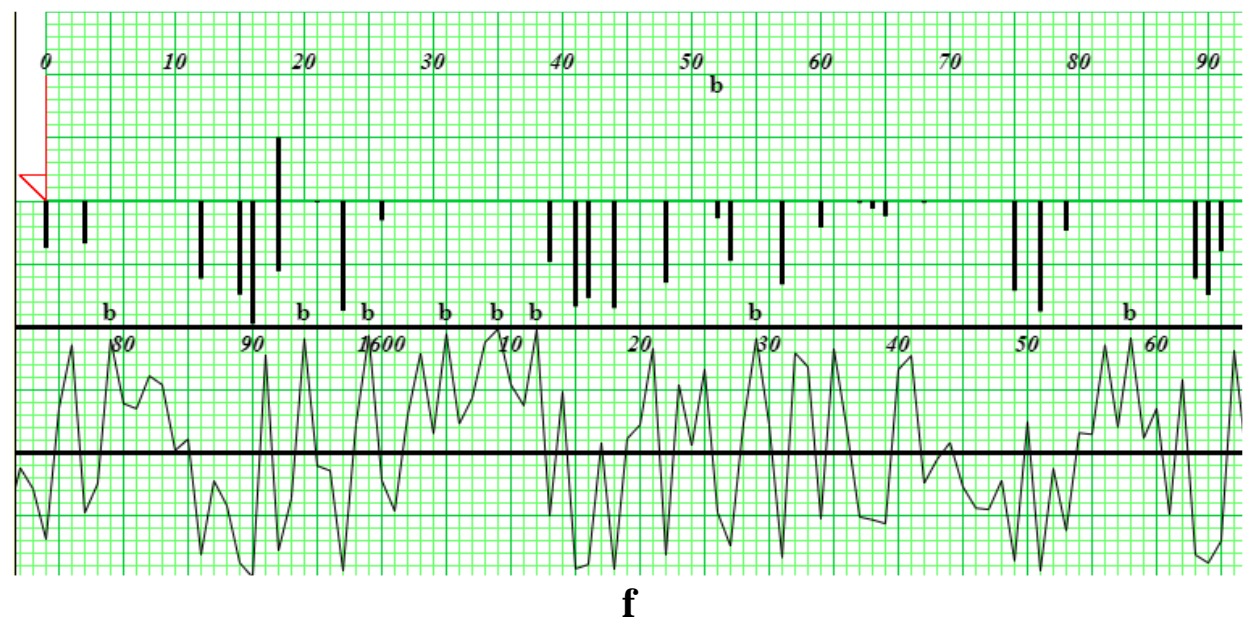




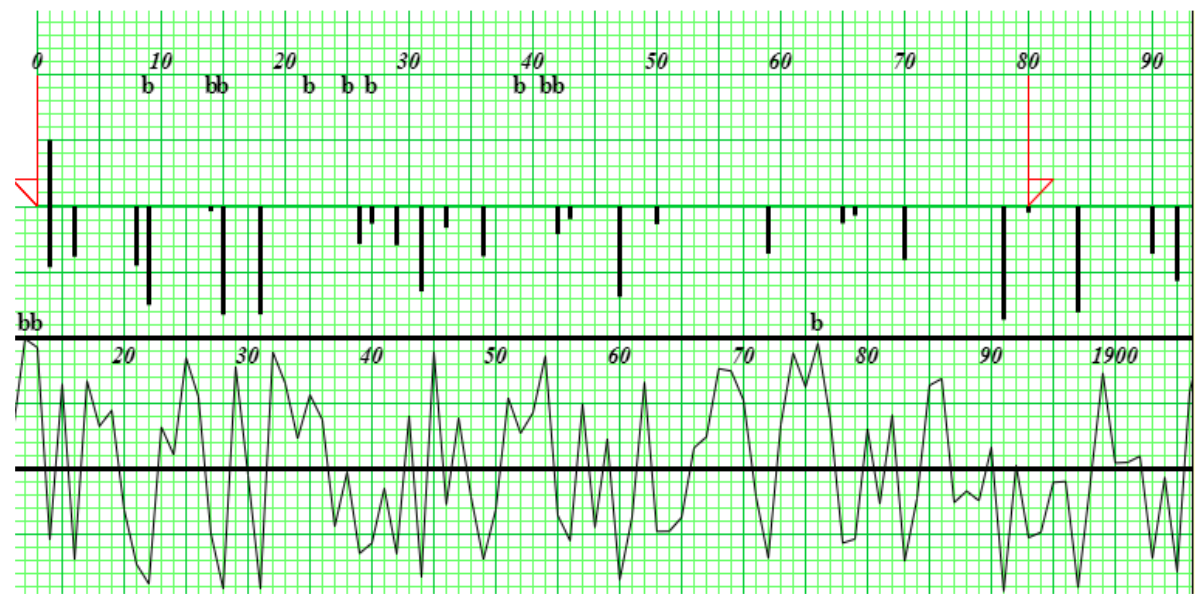

g

TB19’ \& TB20 (1953-2019 AD)

1997, 1998, 2000, 2018.

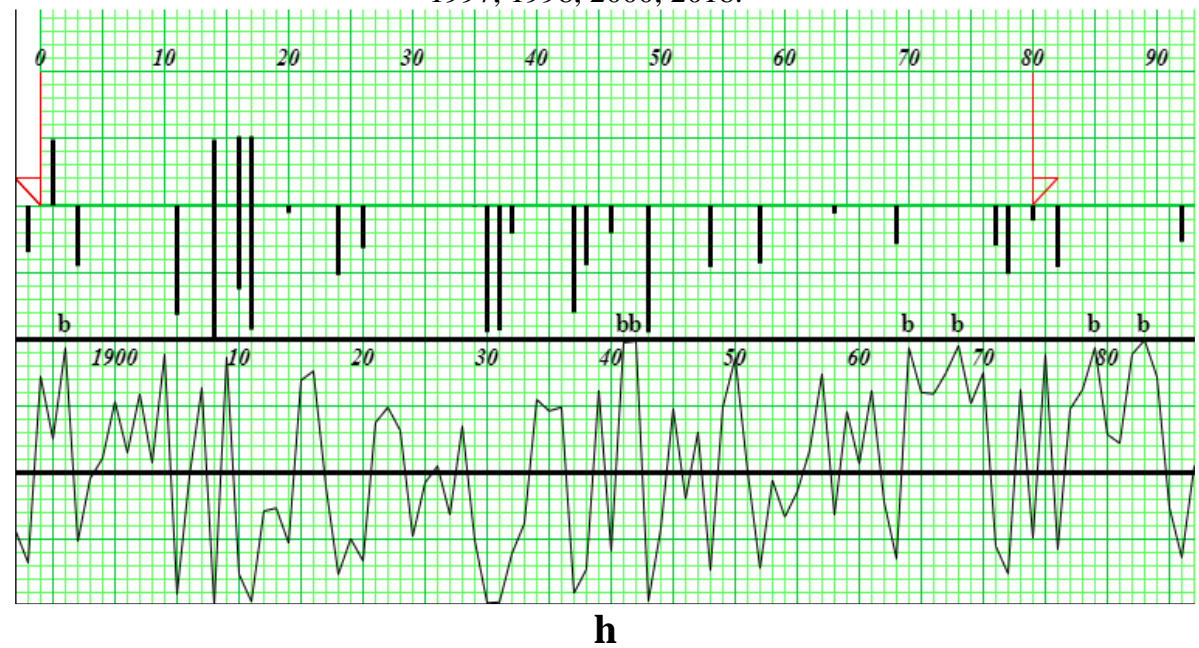

Figure 7. (a-h) Most sensitive growth years of trees, representation through composite skeleton plot and their matching with master skeleton plot and master chronology

\section{Discussion}

Conifers population increases with increase in forest resources and particular species have wide range of distribution. The nature of any forest is determined by interaction of many physical and biological factors resulted in species distribution in particular geographical region depending upon resource requirements in tolerant sites where it makes necessity for survival (Glatzel, 2009; Shaheen et al., 2015). Thus, present study focuses on age and growth rate determination and intra annual fluctuations between different species by graphical representation. In this way, chronology was prepared by Skeleton Plot Model after identification of climate sensitive years in selected species (Sheppard, 2002).

Environmental factors are very important in determining species dominancy at site such as sufficient light, nutrition, moisture and other edapho climate features. So, $A$. pindrow was found to be in large number as edapho climate features favored its growth 
while $T$. baccata, $P$. roxburghii, $C$. deodara was found to be minimal which may due to some anthropogenic activities like cutting, logging, fire and overgrazing.

\section{Age and growth rate studies}

Maximum and minimum age and growth rate of all selected conifers is presented in Table 1. Age and growth rate varies with elevation variation, from species to species and even among the similar species of similar sites. Many researchers have been engaged and are investigating dendrochronological potential from Pakistan and around the globe. Ahmed et al. (1990) reported age of $P$. wallichiana, 112 years with $20.5 \mathrm{~cm}$ dbh from dry temperate area of Takhtesuleman and moist temperate area like Khanaspur, Ayubia and Murree with different diameters, $58 \mathrm{~cm}, 65 \mathrm{~cm}, 58 \mathrm{~cm} \mathrm{dbh}$. A. pindrow showed an age of 103 years and 105 years with 71.2 and $92 \mathrm{~cm}$ dbh respectively from Murree site. An oldest A. pindrow was recorded with age of 277 years and $89 \mathrm{~cm}$ diameter from Ayubia. $C$. deodara was found to be oldest with age of 533 years and $180 \mathrm{~cm}$ dbh from Chitral site. The present study revealed the dominancy of $A$. pindrow with maximum age of 698 years and $149.3 \mathrm{~cm}$ diameter from Kalam Forest Division. Some other researchers from other countries determined dendrochronological potential of trees. Mccarthy and Weetman (2006) determined age of Abies balsames with 264 years black and white spruce with 264 and 247 years respectively from Canada. Muhammad et al. (2019) determined age of $C$. deodara from Kashmir Point Murree, Pakistan. Moreover, age of Pondesera was also determined, 618, 613 and 330 years from different sites of California by Youngblood et al. (2004). In the present study, we determined age of A. pindrow, T. baccata and $P$. roxburghii, 698 years with $149.3 \mathrm{~cm}$ dbh, 479 years with $137.16 \mathrm{~cm}$ and 218 years with $48.76 \mathrm{~cm}$ dbh respectively. $C$. deodara was found in minimum quantity due to reduced competition among trees and illegal cutting and burning of trees. So, significant positive correlation was observed in age and dbh among different species. Age may increase or decrease with increase of diameter as species lie in different geographical positions. Moreover, it was not observed an effective parameter for age variation as regional climate also favors the growth of conifers after Scipioni et al. (2021) and Ahmed et al. (2011).

Maximum and minimum growth rate of all species is presented in Table 1. Growth rate of conifers is also affected by availability of forest resources such as moisture content, favorable temperature and precipitation, adequate nutrients and prevention from natural or unnatural disasters after Ahmed et al. (2012). Among selected species $T$. baccata was found to be with maximum growth rate as environmental factors favored its growth and development. However, growth rate of rest species was also observed reasonable but not responded like T. baccata. Siddiqui et al. (2013) observed slow growth rate of A. pindrow from moist temperate area. Ahmed and Sarangzai (1992) determined growth rate of $P$. wallichiana, $2.5 \mathrm{~cm} /$ years from Murree. Ahmed et al. (2009) also determined as $1.7 \mathrm{~cm} /$ years in $P$. wallichiana from Dir. A. pindrow and $C$. deodara was observed with faster growth rate from Dir and Naran areas. In present study, $T$. baccata was observed with maximum growth rate, $0.66 \mathrm{~cm} / \mathrm{year}$ while it was 0.05 and $0.10 \mathrm{~cm} /$ year as maximum in A. pindrow and $P$. roxburghii respectively.

\section{Correlations study}

Many researchers observed significant positive correlation between age and diameter from different sites of world. Ahmed and Sarangzai (1991) obtained such positive correlation in all selected species. However, Ahmed et al. (1990) did not find significant 
relationship between these parameters in Juniperus excela from Baluchistan, Pakisan. Moreover, Wahab et al. (2008) did not observe such positive relationship in Picea smithiana. Ahmed et al. (2009) did not find such correlation four species i.e., A. pindrow, $P$. wallichiana, $C$. deodara and Picea smithiana but they observed positive relation in $P$. roxburghii. In the present study, A. pindrow, $T$. baccata and $C$. deodara showed positive correlation while $P$. roxburghii showed highly negative correlation between age and diameter.

Growth rate was also observed non-significant by various researches. Muhammad et al. (2021) did not observe significant correlation between dbh and growth rate in $C$. deodara from Murree, Pakistan. Wahab et al. (2008) also observed negative relationship between $\mathrm{dbh}$ and growth rate in $P$. smithiana from conifers of Afghanistan Forest. A. pindrow, $P$. wallichiana and $C$. deodara also showed highly significant negative correlation between dbh and growth rate by Siddiqui et al., 2013. Ahmed et al. (2012) observed strongest response by rainfall. All selected species showed highly negative correlation between dbh and growth rate in present findings as shown in Figures $4 b, 5 b, 6 b$.

\section{Conclusion}

Kalam Valley, located at the junction of three mountains (Hindukush, Himalaya, and Karakoram), has a special topography, according to the researchers. The alpine peaks in the area vary in elevation from 1900 to $4600 \mathrm{~m}$. Conifers grow taller as a result of edaphoclimate factors such as harsh winters, acidic soil, altitude variation, salts, sand, silt, and organic matter. Briefly to conclude, the study site was densely covered with $A$. pindrow as huge number of this species was observed while $C$. deodara was observed as minimal due to some anthropogenic disturbances. Significant positive correlation was observed between age and diameter in all species except $P$. roxburghii after Ahmed and Sarangzai (1991) while no such significant relationship was observed between diameter and growth rate after Wahab et al. (2008). Moreover, environmental factors mainly temperature, precipitation and rainfall also favored age and growth rate of these conifers and $T$. baccata responded well to these climatic variations as maximum growth rate was recorded in this species after Ahmed et al. (2012). A lot of reasons (deforestation, burning of wood as to fulfil needs of coal, overgrazing, town planning, and frequent forest fires) have placed the study site under biological stress due to $C$. deodara was observed a few in number. The chronology presented in results clearly depicted highly sensitive tree rings which were distinct indications of growth rate variations during development of trees. The developed chronologies signify the sensitivity of conifers of dry temperate study region towards diverse climatic factors and could be analyzed to predict future seasonal climate variations. Some highly recommended steps can be followed to conserve species habitat (natural biodiversity) through traditional practices such as:

- Need of awareness and management of forests to regain their valuable potential regarding to scientific research projects.

- Implementation of Forest rules to avoid from illegal cutting, burning and chopping by providing alternative fuel resources.

- Old age trees of study site such as A. pindrow, $T$. baccata, particularly $C$. deodara (national tree of Pakistan) etc. must be declared as cultural heritage of country.

- Ecotourism must be developed to promote cultural and economic significance by working with international counterparts, to acquire global exposure. 
Acknowledgements. Authors are very grateful to Mr. Naveed Shehzad, Hassan Nawaz and Amir Ali for help in Field work and all anonymous referees for their valuable comments and suggestions that have significantly increased the quality of manuscript.

\section{REFERENCES}

[1] Ahmed, M. (2014): The Science of Tree Rings: Dendrochronology. - Qureshi Art Press, Nazimabad, Karachi, pp. 95-108.

[2] Ahmed, M., Sarangzai, A. M. (1991): Dendrochronological approach to estimate age and growth rate of various species from Himalayan region of Pakistan. - Pakistan Journal of Botany 23(1): 78-89.

[3] Ahmed, M., Sarangzai, A. M. (1992): Dendrochronological potential of a few tree species from Himalayan region of Pakistan, a preliminary investigation. - Pakistan Journal of Pure and Applied Sciences 11(2): 65-72.

[4] Ahmed, M., Naqvi, S. H. (2005): Tree ring chronologies of Picea smithiana (Wall) Boiss, and its quantitative vegetation description from Himalayan region of Pakistan. - Pakistan Journal Botany 37(3): 697-707.

[5] Ahmed, M., Nagi, E. E., Wang, E. L. M. (1990): Present state of Juniper in Rodhmallazi forest of Baluchistan. Pakistan. - Pakistan Journal of Forestry 227-236.

[6] Ahmed, M., Wahab, M., Khan, N., Siddiqui, M. F., Khan, M. U., Hussain, S. T. (2009): Age and growth rates of some gymnosperms in Pakistan. A dendrochronological approach. - Pakistan Journal of Botany 41(2): 849-860.

[7] Ahmed, M., Palmer, J., Khan, N., Wahab, M., Fenrick, P., Esper, J., Cook, E. (2011): The dendroclimatic potential of conifers from Northern Pakistan. - Dendrochronologia 29(2): $77-88$.

[8] Ahmed, M., Khan, N., Wahab, M., Zafar, M. U., Palmer, J. (2012): Climate/growth correlations of tree species in the Indus Basin of the Karakoram range, north Pakistan. International Association of Wood Anatomists 33(1) 51-56.

[9] Ali, M., Asad, F., Zhu, H., Ahmed, M., Sigdel, S. R., Huang, R., Sharma, S., Liang, E., Hussain, I., Yaseen, T. (2021): Dendrochronological investigation of selected conifers from Karakoram-Himalaya, Northern Pakistan. - Pakistan Journal of Botany 53(3) 10911099.

[10] Bajwa, G. A., Shahzad, M. K., Satti, H. K. (2015): Climate change and its impacts on growth of blue pine (Pinus wallichiana) in Murree forest division, Pakistan. - Science Technology and Development 34(1): 27-34.

[11] Cook, E. R., Kairiukstis, L. A. (2010): Methods of Dendrochronology: Applications in the Environmental Sciences. - Kluwer Academic Publishers, Dordrecht.

[12] Copenheaver, C. A., Pokorski, E. A., Currie, J. E., Abrams, M. D. (2006): Causation of false ring formation in Pinus banksiana: a comparison of age, canopy class, climate and growth rate. - Forest Ecology and Management 236: 348-355.

[13] Glatzel, G. (2009): Mountain forests in a changing World, an Epilogue. - Mountain Research and Development 29: 188-190.

[14] Gosling, S. N., Lowe, J. A., McGregor, G. R., Pelling, M., Lalamud, B. D. (2009): Association between elevated atmospheric temperature and human mortality: a critical review of the literature. - Climate Change 92: 299-341.

[15] Hart, J. L., Grissino-Mayer, H. D. (2008): Vegetation patterns and dendroecology of a mixed hard wood forest on the Cumberland Plateau: implications for stand development. - Forest Ecology and Management 225: 1960-1975.

[16] Iqbal, H. (2014): Divisional Profile of Kalam Forest Division. - Kalam Forest Division, Madyan Swat.

[17] Khan, N. (2011): Vegetation ecology and dendrochronology of Chitral. - Ph.D Thesis, Department of Botany, Federal Urdu University, Gulshan-e-Iqbal, Karachi. 
[18] Khan, N., Ahmed, M., Wahab, M. (2008): Dendrochronological potential of Picea smithiana (Wall) Boiss. from Afghanistan. - Pakistan Journal of Botany 40(3): 10631070.

[19] Khan, N., Ahmed, M., Shahid, S. S. (2013): Climatic signal in Tree ring chronologies of Cedrus deodara from Chitral Hindukush range of Pakistan. - Geochronometria 40(3): 195-207.

[20] McCarthy, J. W., Weetman, G. (2006): Age and size structure of gap-dynamics, oldgrowth boreal stands in Newfoundland. - Silva Fennica 40: 209-230.

[21] Muhammad, S., Tayyab, M., Khan, Z., Malik, S. M., Akram, N. (2019): Age and growth rate studies of Cedrus deodara (Roxb. ex D. Don) around Kashmir point Reserve Forest of Tehsil Murree. - International Journal of Biosciences 15(3): 458-465.

[22] Muhammad, S., Hasnain, M., Tayyab, M., Khan, Z., Rasool, K. (2021): Dendrochronological potential of blue pine (Pinus wallichiana A.B. Jacks.) of Kuldana Reserve Forest of Tehsil Murree, Pakistan. - Pakistan Journal of Science 73(1): 130-137.

[23] Nash, S. E. (2002): Archeological tree ring dating at the millennium. - Journal of Archeological Research 10(3): 243-266.

[24] Palmer, J., Ahmed, M., Khan, Z. (2011): Application of tree-ring research in Pakistan. Federal Urdu University of Arts, Science and Technology Journal of Biology 1(1): 19-25.

[25] Panyushkina, I. P. (2011): Dendrochronology. McGraw Hill Encyclopedia of Science \& Technology, $11^{\text {th }}$ Ed. - McGraw Hill, New York, pp. 1-11.

[26] Phipps, R. L. (1985): Collecting, Preparing, Crossdating and Measuring Tree Increment Cores. - U.S. Geological Survey, Washington, DC, pp. 35-40.

[27] Scipioni, M. C., Fontana, C., Oliveira, J. M., Junior, L. S., Roig, F. A., Tomazello-Filho, M. (2021): Effects of cold conditions on the growth rates of a subtropical conifer. Dendrochronologia 68: 125858.

[28] Shaffer, G., Olsen, S. M., Pederson, G. O. P. (2009): Long term ocean oxygen depletion in response to carbon dioxide emissions from fossil fuels. - Nature Geoscience 2: 105109.

[29] Shah, H., Jehan, N., Rehman, S. S., Bukhari, S. S. B. (2019): Comparative study of climate change and its impact on ring-widths of spruce (Picea smithiana) at Kalam and Kaghan Forest Divisions, Khyber Pakhtunkhwa, Pakistan. - Sarhad Journal of Agriculture 35(3): 788-797.

[30] Shaheen, H., Sarwar, R., Firdous, S. S., Dar, M. E., Ullah, Z., Khan, S. M. (2015): Distribution and structure of conifers with special emphasis on Taxus baccata in moist temperate Forests of Kashmir Himalayas. - Pakistan Journal of Botany 47(SI): 71-76.

[31] Sheppard, P. R. (2002): Web-based tools for teaching dendrochronology. - Journal of Natural Resources and Life Sciences Education 31: 123-129.

[32] Siddiqui, M. F., Shaukat, S. S., Ahmed, M., Khan, N., Khan, I. A. (2013): Vegetation environment relationship of conifer dominating forests of moist temperate belt of Himalayan and Hindukush regions of Pakistan. - Pakistan Journal of Botany 45: 577592.

[33] Speer, J. H. (2010): Fundamentals of Tree Ring Research. - University of Arizona Press, Tucson.

[34] Sun, Y., Wang, L., Yin, H. (2016): Influence of climatic factors on tree-ring maximum latewood density of Picea schrenkiana in Xinjiang, China. - Frontiers in Earth Science 10(1): 126-134.

[35] Vasconcellos, T. J. M., Filho, T., Callado, C. H. (2019): Dendrochronology and Dendroclimatology of Ceiba apeciosa (A. St. Hil) Ravenna (Malvaceaea) exposed to urban pollution in Rio de Jeneiro city, Brazil. - Dendrochronologia 53: 104-113.

[36] Volney, W. J. A., Mallet, K. I. (1992): Light rings and the age of jack pine trees. Canadian Journal of Forest Research 22: 2011-2013.

[37] Wahab, M. (2011): Population dynamics and dendrochronological potential of pine tree species from District Dir. - Ph.D. thesis. Department of Botany, Federal Urdu University, Gulshan-e-Iqbal, Karachi. 


$$
-4419 \text { - }
$$

[38] Wahab, M., Ahmed, M., Khan, N. (2008): Phytosociology and dynamics of some pine forests of Afghanistan. - Pakistan Journal of Botany 40: 1071-1079.

[39] Yamaguchi, D. K. (1991): A simple method for cross-dating increment cores from living trees. - Canadian Journal of Forest Research 21: 414-416.

[40] Youngblood, A., Max, T., Coe. K. (2004): Stand structure in east side old-growth forests of Oregon and northern California. - Forest Ecology and Management 199: 191-217. 